\title{
Analysis of a stochastic cooperation-competition model
}

\author{
Weidong Zhou' ${ }^{1}$ Zejing Xing ${ }^{1 *}$, Hongtao Cui ${ }^{2}$ and Ye Li ${ }^{3}$
}

${ }^{\text {*Correspondence: }}$

xingzejing1140@163.com

${ }^{1}$ College of Automation, Harbin

Engineering University, Harbin,

China

Full list of author information is

available at the end of the article

\begin{abstract}
In this paper, we explore dynamic properties of a stochastic cooperation-competition model. Some sufficient conditions are established for stochastic persistence and stochastic extinction of species. Studies suggest that the noise may have a positive effect on the persistence of species. We also analyze global asymptotic stability of positive solutions and give a stationary distribution of this stochastic model which has the ergodic property. Finally, some numerical simulations are presented to illustrate or complement our mathematical findings.
\end{abstract}

Keywords: stochastic cooperation-competition model; stochastic persistence and stochastic extinction; global asymptotic stability; stationary distribution

\section{Introduction}

There is already growing evidence that the ecological relationship in the population is a significant global driver of biodiversity change and decline. Competition and mutualism are the two basic elements of interspecific relationship, and they are an important driving force in structuring ecological communities. The cooperation-competition model describes that two different populations exist in a relationship in which each individual benefits from the activity of the other and are both in competition with a third population. There exist some excellent results to explore the qualitative and stability properties of the cooperation-competition model [1-6]. On the other hand, it is recognized that there is a large number of random factors in the real natural environment. These random factors of the environment are not only an integral part of any realistic ecosystem, but they also may lead to complete extinction of populations. The existing research works have investigated dynamic properties of stochastic predator-prey models [7-12], stochastic competitive models [13-18], stochastic mutualism models [19-24] and stochastic three-species models [25-33]. To the best of our knowledge, there are few studies to analyze the dynamics of a stochastic cooperation-competition model.

In the present paper, our model is based on the following deterministic cooperationcompetition model:

$$
\begin{aligned}
& d x(t)=x(t)\left(r_{1}-a_{11} x(t)+a_{12} y(t)-a_{13} z(t)\right), \\
& d y(t)=y(t)\left(r_{2}+a_{21} x(t)-a_{22} y(t)-a_{23} z(t)\right), \\
& d z(t)=z(t)\left(r_{3}-a_{31} x(t)-a_{32} y(t)-a_{33} z(t)\right),
\end{aligned}
$$

(C) The Author(s) 2018. This article is distributed under the terms of the Creative Commons Attribution 4.0 International License (http://creativecommons.org/licenses/by/4.0/), which permits unrestricted use, distribution, and reproduction in any medium, provided you give appropriate credit to the original author(s) and the source, provide a link to the Creative Commons license, and indicate if changes were made. 
where $x(t), y(t)$ and $z(t)$ are the densities of species, $r_{i}(i=1,2,3)$ is the per capita growth rate of species, $a_{i i}(i=1,2,3)$ is the intraspecific competition rate of species, $a_{12}$ and $a_{21}$ are interspecific mutual rates of species, $a_{i 3}$ and $a_{3 i}(i=1,2)$ are interspecific competitive rates of species. Here we assume that all the parameters involved in the model are positive, and the environmental fluctuation modelled by means of independent Gaussian white noises mainly influences the per capita growth rate $r_{i}(i=1,2,3)$ of species since May [34] have pointed out that the per capita growth rate exhibits random fluctuation to a greater or lesser extent (also see [35, 36]). Let $L(t)=(x(t), y(t), z(t))^{T}$ be a Markov process with the following specifications:

$$
\begin{aligned}
& E[x(t+\Delta t)-x(t) \mid L(t)=l] \approx x\left(r_{1}-a_{11} x+a_{12} y-a_{13} z\right) \Delta t, \\
& E[y(t+\Delta t)-y(t) \mid L(t)=l] \approx y\left(r_{2}+a_{21} x-a_{22} y-a_{23} z\right) \Delta t, \\
& E[z(t+\Delta t)-z(t) \mid L(t)=l] \approx z\left(r_{3}-a_{31} x-a_{32} y-a_{33} z\right) \Delta t,
\end{aligned}
$$

and

$$
\begin{aligned}
& \operatorname{Var}[x(t+\Delta t)-x(t) \mid L(t)=l] \approx a_{1}^{2} x^{2} \Delta t, \\
& \operatorname{Var}[y(t+\Delta t)-y(t) \mid L(t)=l] \approx a_{2}^{2} y^{2} \Delta t, \\
& \operatorname{Var}[z(t+\Delta t)-z(t) \mid L(t)=l] \approx a_{3}^{2} z^{2} \Delta t,
\end{aligned}
$$

for sufficiently small $\Delta t$ (see [37]). Then we consider the following stochastic cooperationcompetition model of Lotka-Volterra type:

$$
\begin{aligned}
& d x(t)=x(t)\left(r_{1}-a_{11} x(t)+a_{12} y(t)-a_{13} z(t)\right)+\alpha_{1} x(t) d B_{1}(t), \\
& d y(t)=y(t)\left(r_{2}+a_{21} x(t)-a_{22} y(t)-a_{23} z(t)\right)+\alpha_{2} y(t) d B_{2}(t), \\
& d z(t)=z(t)\left(r_{3}-a_{31} x(t)-a_{32} y(t)-a_{33} z(t)\right)+\alpha_{3} z(t) d B_{3}(t),
\end{aligned}
$$

where $\left(B_{1}(t), B_{2}(t), B_{3}(t)\right)^{T}$ is a three-dimensional Brownian motion defined on a complete probability space $(\Omega, F, P), \alpha_{i}^{2}(i=1,2,3)$ is the intensity of the white noise.

The paper focuses on two main aspects of the stochastic cooperation-competition model (1.2). One is to establish stochastic persistence and extinction of species and sufficient conditions for global asymptotic stability of the positive solutions of model (1.2). Especially, we investigate the important effect of the noise on species. Another aspect is to prove the existence of stationary distribution of model (1.2) and the fact that there exists the ergodic property in the stationary distribution. Here, if a stationary distribution has the ergodic property, then this means that the mean of population density in time with the development of time is equal to the mean of population density in space.

This work is organized as follows. In the next section, we state our main results: stochastic persistence and stochastic extinction; global asymptotic stability of positive solutions; and the existence of stationary distribution. In Section 3, we give the rigorous proofs of the main results. In Section 4, we do some numerical simulations to illustrate or complement our mathematical findings. The final section summarizes our findings. 


\section{Main results}

Three main results are obtained, including (1) stochastic persistence and stochastic extinction; (2) global asymptotic stability of positive solutions; and (3) the existence of stationary distribution. We will achieve the three results through several steps.

At the beginning, we introduce some notations. Let

$$
\begin{aligned}
& {[f]=\frac{1}{t} \int_{0}^{t} f(s) d s} \\
& {[f]^{*}=\limsup _{t \rightarrow+\infty} \frac{1}{t} \int_{0}^{t} f(s) d s,} \\
& {[f]_{*}=\liminf _{t \rightarrow+\infty} \frac{1}{t} \int_{0}^{t} f(s) d s,} \\
& \rho_{1}=a_{22} r_{1}+a_{12} r_{2}, \quad \rho_{2}=a_{21} r_{1}+a_{11} r_{2}, \quad \rho_{3}=a_{33} r_{1}+a_{13} r_{3}, \\
& \rho_{4}=a_{11} r_{3}-a_{31} r_{1}, \quad \rho_{5}=a_{33} r_{2}+a_{23} r_{3}, \quad \rho_{6}=a_{22} r_{3}+a_{32} r_{2}, \\
& \tilde{\rho}_{1}=a_{22} \alpha_{1}^{2} / 2+a_{12} \alpha_{2}^{2} / 2, \quad \tilde{\rho}_{2}=a_{21} \alpha_{1}^{2} / 2+a_{11} \alpha_{2}^{2} / 2, \\
& \tilde{\rho}_{3}=a_{33} \alpha_{1}^{2} / 2+a_{13} \alpha_{3}^{2} / 2, \quad \tilde{\rho}_{4}=a_{11} \alpha_{3}^{2} / 2-a_{31} \alpha_{1}^{2} / 2, \\
& \tilde{\rho}_{5}=a_{33} \alpha_{2}^{2} / 2-a_{23} \alpha_{3}^{2} / 2, \quad \tilde{\rho}_{6}=a_{22} \alpha_{3}^{2} / 2-a_{32} \alpha_{2}^{2} / 2 \text {, } \\
& G=\left|\begin{array}{ccc}
a_{11} & -a_{12} & a_{13} \\
-a_{21} & a_{22} & a_{23} \\
a_{31} & a_{32} & a_{33}
\end{array}\right|, \quad G_{1}=\left|\begin{array}{ccc}
r_{1} & -a_{12} & a_{13} \\
r_{2} & a_{22} & a_{23} \\
r_{3} & a_{32} & a_{33}
\end{array}\right|, \quad G_{2}=\left|\begin{array}{ccc}
a_{11} & r_{1} & a_{13} \\
-a_{21} & r_{2} & a_{23} \\
a_{31} & r_{3} & a_{33}
\end{array}\right|, \\
& G_{3}=\left|\begin{array}{ccc}
a_{11} & -a_{12} & r_{1} \\
-a_{21} & a_{22} & r_{2} \\
a_{31} & a_{32} & r_{3}
\end{array}\right|, \quad \tilde{G}_{1}=\left|\begin{array}{ccc}
\alpha_{1}^{2} / 2 & -a_{12} & a_{13} \\
\alpha_{2}^{2} / 2 & a_{22} & a_{23} \\
\alpha_{3}^{2} / 2 & a_{32} & a_{33}
\end{array}\right|, \quad \tilde{G}_{2}=\left|\begin{array}{ccc}
a_{11} & \alpha_{1}^{2} / 2 & a_{13} \\
-a_{21} & \alpha_{2}^{2} / 2 & a_{23} \\
a_{31} & \alpha_{3}^{2} / 2 & a_{33}
\end{array}\right|, \\
& \tilde{G}_{3}=\left|\begin{array}{ccc}
a_{11} & -a_{12} & \alpha_{1}^{2} / 2 \\
-a_{21} & a_{22} & \alpha_{2}^{2} / 2 \\
a_{31} & a_{32} & \alpha_{3}^{3} / 2
\end{array}\right| \text {. }
\end{aligned}
$$

Theorem 2.1 For any given initial value $(x(0), y(0), z(0))^{T} \in \mathbb{R}_{+}^{3}$, there exists a unique solution $(x(t), y(t), z(t))^{T}$ of $(1.2)$ on $t \geq 0$, and this solution will remain in $\mathbb{R}_{+}^{3}$ with probability 1 .

Proof It is clear that, for any given initial value $(x(0), y(0), z(0))^{T} \in \mathbb{R}_{+}^{3}$, there exists a unique local solution $(x(t), y(t), z(t))^{T}$ of $(1.2)$ on $t \in\left[0, t_{e}\right)$, where $t_{e}$ is the explosion time. Let $k_{0}>0$ be sufficiently large such that any given initial value $(x(t), y(t), z(t))^{T}$ lies within $\left[1 / k_{0}, k_{0}\right] \times$ $\left[1 / k_{0}, k_{0}\right] \times\left[1 / k_{0}, k_{0}\right]$. For each integer $k>k_{0}$, define the stopping time.

$$
\tau_{k}=\inf \left\{t \in\left[0, t_{e}\right): x(t) \notin(1 / k, k) \text { or } y(t) \notin(1 / k, k) \text { or } z(t) \notin(1 / k, k)\right\} .
$$

Carrying out similar arguments of Theorem 2.1 in [38], we conclude that $\tau_{\infty}>\infty$ a.s. where $\tau_{\infty}=\lim _{k \rightarrow \infty} \tau_{k}$. Here we omit some details.

The first part of our results solves stochastic persistence of the species in model (1.2).

Definition 2.1 (see [25]) Species $N(t)$ is said to be stochastically persistent in the mean if $[N]_{*}>0$. 
It is noteworthy that stochastic persistence for the stochastic population model has many forms of definition; for example, stochastic persistence defined in the form of distribution, or stochastic persistence defined in the form of expectation. Here stochastic persistence is defined as the mean of the sample paths of the solution in time.

Theorem 2.2 If $a_{11} a_{22}>a_{12} a_{21}$ and $r_{i}<\alpha_{i}^{2} / 2$ for $i=1,2,3$, then all the species of model (1.2) go to extinction a.s.

Theorem 2.3 Let $G>0, a_{11}>a_{12}$ and $a_{22}>a_{21}$. Then

(i) if $r_{1}>\alpha_{1}^{2} / 2, \rho_{2}<\tilde{\rho}_{2}, G_{3}<\tilde{G}_{3}$, then the species $y(t)$ and $z(t)$ go to extinction a.s. and $x(t)$ is stochastically persistent with

$$
\lim _{t \rightarrow+\infty} \frac{1}{t} \int_{0}^{t} x(s) d s=\frac{r_{1}-\alpha_{1}^{2} / 2}{a_{11}} \quad \text { a.s. }
$$

(ii) if $r_{2}>\alpha_{2}^{2} / 2, \rho_{1}<\tilde{\rho}_{1}, G_{3}<\tilde{G}_{3}$, then the species $x(t)$ and $z(t)$ go to extinction a.s. and $y(t)$ is stochastically persistent with

$$
\lim _{t \rightarrow+\infty} \frac{1}{t} \int_{0}^{t} y(s) d s=\frac{r_{2}-\alpha_{2}^{2} / 2}{a_{22}} \text { a.s. }
$$

(iii) if $r_{3}>\alpha_{3}^{2} / 2$ and one of the following conditions holds:

$\left(\mathrm{A}_{1}\right) \rho_{1}<\tilde{\rho}_{1}, \rho_{5}<\tilde{\rho}_{5}$ and $a_{22} a_{33}>a_{23} a_{32}$;

$\left(\mathrm{A}_{2}\right) \rho_{1}<\tilde{\rho}_{1}, \rho_{3}<\tilde{\rho}_{3}$ and $a_{11} a_{33}>a_{13} a_{31}$;

$\left(\mathrm{A}_{3}\right) \rho_{2}<\tilde{\rho}_{2}, \rho_{5}<\tilde{\rho}_{5}$ and $a_{22} a_{33}>a_{23} a_{32}$;

$\left(\mathrm{A}_{4}\right) \rho_{2}<\tilde{\rho}_{2}, \rho_{3}<\tilde{\rho}_{3}$ and $a_{11} a_{33}>a_{13} a_{31}$,

then the species $x(t)$ and $y(t)$ go to extinction a.s. and $z(t)$ is stochastically persistent with

$$
\lim _{t \rightarrow+\infty} \frac{1}{t} \int_{0}^{t} z(s) d s=\frac{r_{3}-\alpha_{3}^{2} / 2}{a_{33}} \text { a.s. }
$$

Theorem 2.4 Let $G>0, a_{11}>a_{12}$ and $a_{22}>a_{21}$. Then

(iv) if $\rho_{1}>\tilde{\rho}_{1}, \rho_{2}>\tilde{\rho}_{2}$ and $G_{3}<\tilde{G}_{3}$, then the species $z(t)$ goes to extinction a.s. and the species $x(t)$ and $y(t)$ are stochastically persistent with

$$
\begin{aligned}
& \lim _{t \rightarrow+\infty} \frac{1}{t} \int_{0}^{t} x(s) d s=\frac{\rho_{1}-\tilde{\rho}_{1}}{a_{11} a_{22}-a_{12} a_{21}} \quad \text { a.s. } \\
& \lim _{t \rightarrow+\infty} \frac{1}{t} \int_{0}^{t} y(s) d s=\frac{\rho_{2}-\tilde{\rho}_{2}}{a_{11} a_{22}-a_{12} a_{21}} \quad \text { a.s. }
\end{aligned}
$$

(v) if $a_{11} a_{33}>a_{13} a_{31}, \rho_{3}>\tilde{\rho}_{3}, \rho_{4}>\tilde{\rho}_{4}$ and $G_{2}<\tilde{G}_{2}$, then the species $y(t)$ goes to extinction a.s. and the species $x(t)$ and $z(t)$ are stochastically persistent with

$$
\begin{aligned}
& \lim _{t \rightarrow+\infty} \frac{1}{t} \int_{0}^{t} x(s) d s=\frac{\rho_{3}-\tilde{\rho}_{3}}{a_{11} a_{33}-a_{13} a_{31}} \quad \text { a.s. } \\
& \lim _{t \rightarrow+\infty} \frac{1}{t} \int_{0}^{t} z(s) d s=\frac{\rho_{4}-\tilde{\rho}_{4}}{a_{11} a_{33}-a_{13} a_{31}} \quad \text { a.s. }
\end{aligned}
$$


(vi) if $a_{22} a_{33}>a_{23} a_{32}, \rho_{5}>r_{5}, \rho_{6}>r_{6}$ and $G_{1}<\tilde{G}_{1}$, then the species $x(t)$ goes to extinction a.s. and the species $y(t)$ and $z(t)$ are stochastically persistent with

$$
\begin{aligned}
& \lim _{t \rightarrow+\infty} \frac{1}{t} \int_{0}^{t} y(s) d s=\frac{\rho_{5}-\tilde{\rho}_{5}}{a_{22} a_{33}-a_{23} a_{32}} \quad \text { a.s. } \\
& \lim _{t \rightarrow+\infty} \frac{1}{t} \int_{0}^{t} z(s) d s=\frac{\rho_{6}-\tilde{\rho}_{6}}{a_{22} a_{33}-a_{23} a_{32}} \quad \text { a.s. }
\end{aligned}
$$

Theorem 2.5 Let $G>0, a_{11}>a_{12}, a_{22}>a_{21}, a_{11} a_{33}>a_{13} a_{31}$ and $a_{22} a_{33}>a_{23} a_{32}$. If $G_{i}>\tilde{G}_{i}$, $i=1,2,3$, then all the species are stochastically persistent with

$$
\begin{aligned}
& \lim _{t \rightarrow+\infty} \frac{1}{t} \int_{0}^{t} x(s) d s=\frac{G_{1}-\tilde{G}_{1}}{G} \quad \text { a.s., } \\
& \lim _{t \rightarrow+\infty} \frac{1}{t} \int_{0}^{t} y(s) d s=\frac{G_{2}-\tilde{G}_{2}}{G} \quad \text { a.s., } \\
& \lim _{t \rightarrow+\infty} \frac{1}{t} \int_{0}^{t} z(s) d s=\frac{G_{3}-\tilde{G}_{3}}{G} \quad \text { a.s. }
\end{aligned}
$$

The second part of our results is to establish global asymptotic stability of the positive solutions of model (1.2).

Definition 2.2 (see [25]) (1.2) is said to be globally asymptotically stable (or globally attractive) if $\lim _{t \rightarrow \infty} \max \left\{\left|x_{1}(t)-x_{2}(t)\right|,\left|y_{1}(t)-y_{2}(t)\right|,\left|z_{1}(t)-z_{2}(t)\right|\right\}=0$ a.s., where $\left(x_{i}(t), y_{i}(t), z_{i}(t)\right), i=1,2$, are two arbitrary solutions of (1.2) with the initial values $\left(x_{i}(0), y_{i}(0), z_{i}(0)\right) \in \mathbb{R}_{+}^{3}, i=1,2$.

Theorem 2.6 If there exists a positive constant $\lambda_{i}, i=1,2,3$, such that

$$
\lambda_{1} a_{11} \geq \lambda_{2} a_{21}+\lambda_{3} a_{31}, \quad \lambda_{2} a_{22} \geq \lambda_{1} a_{12}+\lambda_{3} a_{32}, \quad \lambda_{3} a_{33} \geq \lambda_{1} a_{13}+\lambda_{2} a_{23},
$$

then model (1.2) is globally asymptotically stable.

The final part of our results is to investigate the existence of the stationary distribution of model (1.2) and to prove that this stationary distribution has the ergodic property.

Definition 2.3 (see [39]) The distribution $\mu(s)$ is called stationary if $\mu(s)$ satisfies

$$
\mu(\omega)=\int P(t, s, \omega) d \mu(\omega)
$$

for all $t>0$, where $P(t, s, \omega)$ is a transition probability function.

Let

$$
\begin{aligned}
& \delta_{1}=a_{11}-\left(a_{12}-a_{13}-a_{21}-a_{31}\right) / 2, \\
& \delta_{2}=a_{22}-\left(a_{12}-a_{21}-a_{23}-a_{32}\right) / 2, \\
& \delta_{3}=a_{33}-\left(a_{13}-a_{23}-a_{31}-a_{32}\right) / 2
\end{aligned}
$$


and $\left(x^{*}, y^{*}, z^{*}\right)^{T}$ be the positive equilibrium point of the corresponding deterministic model of model (1.2), that is, $x^{*}=G_{1} / G>0, y^{*}=G_{2} / G>0, z^{*}=G_{3} / G>0$.

Theorem 2.7 If $\delta_{i}>0, i=1,2,3$ and

$$
\left(\alpha_{1}^{2} x^{*}+\alpha_{2}^{2} y^{*}+\alpha_{3}^{2} z^{*}\right) / 2<\min \left\{\delta_{1}\left(x^{*}\right)^{2}, \delta_{2}\left(y^{*}\right)^{2}, \delta_{3}\left(z^{*}\right)^{2}\right\}
$$

then there is a stationary distribution $\mu(\cdot)$ for model (1.2) and it has the ergodic property

$$
\begin{aligned}
& P\left\{\lim _{t \rightarrow+\infty} \frac{1}{t} \int_{0}^{t} x(s) d s=\int_{\mathbb{R}_{+}^{3}} \omega_{1} \mu\left(d \omega_{1}, d \omega_{2}, d \omega_{3}\right)\right\}=1, \\
& P\left\{\lim _{t \rightarrow+\infty} \frac{1}{t} \int_{0}^{t} y(s) d s=\int_{\mathbb{R}_{+}^{3}} \omega_{2} \mu\left(d \omega_{1}, d \omega_{2}, d \omega_{3}\right)\right\}=1, \\
& P\left\{\lim _{t \rightarrow+\infty} \frac{1}{t} \int_{0}^{t} z(s) d s=\int_{\mathbb{R}_{+}^{3}} \omega_{3} \mu\left(d \omega_{1}, d \omega_{2}, d \omega_{3}\right)\right\}=1 .
\end{aligned}
$$

The above theorem shows that if a stationary distribution has the ergodic property, then the mean of population density in time with the development of time is equal to the mean of population density in space with probability one.

\section{Proofs of the main results}

This section presents the proofs of Theorems 2.2-2.7.

\subsection{The proofs of Theorems 2.2-2.5}

In order to complete the proofs of Theorems 2.2-2.5, we first introduce two important lemmas.

Lemma 3.1 (see [25]) Let $z \in C\left(\Omega \times[0,+\infty), \mathbb{R}_{+}\right)$.

(i) If there exist two positive constants $T$ and $\lambda_{0}$ such that

$$
\ln z(t) \leq \lambda t-\lambda_{0} \int_{0}^{t} z(s) d s+\sum_{i=1}^{n} \sigma_{i} B_{i}(t)
$$

for all $t \geq T$, where $B_{i}(t), 1 \leq i \leq n$, are independent standard Brownian motions and $\sigma_{i}, 1 \leq i \leq n$, are constants, then $[z]^{*} \leq \lambda / \lambda_{0}$ a.s. if $\lambda \geq 0$ or $\lim _{t \rightarrow+\infty} z(t)=0$ a.s. if $\lambda<0$.

(ii) If there exist three positive constants $T, \lambda$ and $\lambda_{0}$ such that

$$
\ln z(t) \geq \lambda t-\lambda_{0} \int_{0}^{t} z(s) d s+\sum_{i=1}^{n} \sigma_{i} B_{i}(t)
$$

for all $t \geq T$, where $B_{i}(t), 1 \leq i \leq n$, are independent standard Brownian motions and $\sigma_{i}, 1 \leq i \leq n$, are constants, then $[z]_{*} \geq \lambda / \lambda_{0}$ a.s.

Similar to Lemmas 3.1, 3.4 in [14], Lemma 3.9 in [19] and Theorem 2.5 in [26], we have the following lemma. 
Lemma 3.2 If $a_{11}>a_{12}$ and $a_{22}>a_{21}$, then for any $p \geq 1$ and solution $(x(t), y(t), z(t))^{T} \in \mathbb{R}_{+}^{3}$ of (1.2), there is a constant $K=K(p)$ such that

$$
\limsup _{t \rightarrow \infty} E\left(x(t)^{p}\right) \leq K, \quad \limsup _{t \rightarrow \infty} E\left(y(t)^{p}\right) \leq K, \quad \limsup _{t \rightarrow \infty} E\left(z(t)^{p}\right) \leq K
$$

and

$$
\begin{aligned}
& \limsup _{t \rightarrow \infty} \frac{\ln x(t)}{\ln t} \leq 1 \quad \text { a.s., } \\
& \limsup _{t \rightarrow \infty} \frac{\ln y(t)}{\ln t} \leq 1 \quad \text { a.s., } \\
& \limsup _{t \rightarrow \infty} \frac{\ln z(t)}{\ln t} \leq 1 \quad \text { a.s. }
\end{aligned}
$$

Proof of Theorem 2.2 By applying Itô's formula to model (1.2), we get

$$
\begin{aligned}
& d \ln x(t)=\left[r_{1}-\alpha_{1}^{2} / 2-a_{11} x(t)+a_{12} y(t)-a_{13} z(t)\right] d t+\alpha_{1} d B_{1}(t), \\
& d \ln y(t)=\left[r_{2}-\alpha_{2}^{2} / 2+a_{21} x(t)-a_{22} y(t)-a_{23} z(t)\right] d t+\alpha_{2} d B_{2}(t), \\
& d \ln z(t)=\left[r_{3}-\alpha_{3}^{2} / 2-a_{31} x(t)-a_{32} y(t)-a_{33} z(t)\right] d t+\alpha_{3} d B_{3}(t) .
\end{aligned}
$$

Integrating from 0 to $t$ on both sides of the above equation and dividing by $t$, we obtain

$$
\frac{1}{t} \ln \frac{x(t)}{x(0)}=r_{1}-\frac{\alpha_{1}^{2}}{2}-a_{11}[x(t)]+a_{12}[y(t)]-a_{13}[z(t)]+\frac{\alpha_{1} B_{1}(t)}{t} .
$$

Similarly, we have

$$
\begin{aligned}
& \frac{1}{t} \ln \frac{y(t)}{y(0)}=r_{2}-\frac{\alpha_{2}^{2}}{2}+a_{21}[x(t)]-a_{22}[y(t)]-a_{23}[z(t)]+\frac{\alpha_{2} B_{2}(t)}{t} \\
& \frac{1}{t} \ln \frac{z(t)}{z(0)}=r_{3}-\frac{\alpha_{3}^{2}}{2}-a_{31}[x(t)]-a_{32}[y(t)]-a_{33}[z(t)]+\frac{\alpha_{3} B_{3}(t)}{t} .
\end{aligned}
$$

A direct calculation shows that

$$
\frac{1}{t} \ln \frac{z(t)}{z(0)} \leq r_{3}-\frac{\alpha_{3}^{2}}{2}-a_{33}[z(t)]+\frac{\alpha_{3} B_{3}(t)}{t} .
$$

By Lemma 3.1, we have $\lim _{t \rightarrow+\infty} z(t)=0$ a.s. if $r_{3}<\alpha_{3}^{2} / 2$ holds. It follows from $a_{11} a_{22}>$ $a_{12} a_{21}$ that there are positive constants $c_{1}$ and $d_{1}$ such that $a_{22} / a_{12} \geq c_{1} / d_{1} \geq a_{21} / a_{11}$. Equation (3.2) multiplied by $c_{1}$ plus equation (3.3) multiplied by $d_{1}$ gives

$$
\begin{aligned}
c_{1} \frac{1}{t} \ln \frac{x(t)}{x(0)}+d_{1} \frac{1}{t} \ln \frac{y(t)}{y(0)}= & \frac{1}{t} \ln \frac{x(t)^{c_{1}} y(t)^{d_{1}}}{x(0)^{c_{1}} y(0)^{d_{1}}} \\
= & c_{1}\left(r_{1}-\alpha_{1}^{2} / 2\right)+d_{1}\left(r_{2}-\alpha_{2}^{2} / 2\right)-\left(c_{1} a_{11}-d_{1} a_{21}\right)[x(t)] \\
& -\left(d_{1} a_{22}-c_{1} a_{12}\right)[y(t)]-\left(c_{1} a_{13}+d_{1} a_{23}\right)[z(t)] \\
& +c_{1} \alpha_{1} B_{1}(t) / t+d_{1} \alpha_{2} B_{2}(t) / t .
\end{aligned}
$$


In view of $r_{i}<\alpha_{i}^{2} / 2, i=1,2, c_{1} a_{11}-d_{1} a_{21} \geq 0$ and $d_{1} a_{22}-c_{1} a_{12} \geq 0$, we obtain $\lim _{t \rightarrow+\infty} x(t)^{c_{1}} y(t)^{d_{1}}=0$, a.s. Then there are two cases:

$$
\lim _{t \rightarrow+\infty} x(t)=0 \quad \text { a.s. }
$$

or

$$
\lim _{t \rightarrow+\infty} y(t)=0 \quad \text { a.s. }
$$

If (3.7) holds, substituting (3.7) into (3.3), we have

$$
\frac{1}{t} \ln \frac{y(t)}{y(0)} \leq r_{2}-\frac{\alpha_{2}^{2}}{2}+\varepsilon-a_{22}[y(t)]+\frac{\alpha_{2} B_{2}(t)}{t} .
$$

It follows from Lemma 3.1 that $\lim _{t \rightarrow+\infty} y(t)=0$ a.s. if $\varepsilon$ is sufficiently small such that $r_{2}-\alpha_{2}^{2} / 2+\varepsilon<0$. Similarly, we conclude that $\lim _{t \rightarrow+\infty} x(t)=0$ a.s. if (3.8) holds. The proof of Theorem 2.2 is complete.

Proof of Theorem 2.3 (i) Let

$$
c_{2}=\left(a_{21} a_{32}+a_{22} a_{31}\right) /\left(a_{11} a_{22}-a_{12} a_{21}\right), \quad d_{2}=\left(a_{12} a_{31}+a_{11} a_{32}\right) /\left(a_{11} a_{22}-a_{12} a_{21}\right) .
$$

By multiplying both sides of (3.2), (3.3) and (3.4) by $c_{2}, d_{2}$ and -1 , respectively, and then adding these three equalities, we get

$$
\begin{aligned}
\frac{1}{t} \ln \frac{z(t)}{z(0)}= & \frac{c_{2}}{t} \ln \frac{x(t)}{x(0)}+\frac{d_{2}}{t} \ln \frac{y(t)}{y(0)}+\frac{G_{3}-\tilde{G}_{3}}{a_{11} a_{22}-a_{12} a_{21}}-\frac{G}{a_{11} a_{22}-a_{12} a_{21}}[z(t)] \\
& +\frac{\alpha_{3} B_{3}(t)-\alpha_{1} c_{2} B_{1}(t)-\alpha_{2} d_{2} B_{2}(t)}{t} \\
\leq & \frac{G_{3}-\tilde{G}_{3}}{a_{11} a_{22}-a_{12} a_{21}}+\varepsilon-\frac{G}{a_{11} a_{22}-a_{12} a_{21}}[z(t)] \\
& +\frac{\alpha_{3} B_{3}(t)-\alpha_{1} c_{2} B_{1}(t)-\alpha_{2} d_{2} B_{2}(t)}{t}
\end{aligned}
$$

for sufficiently large $t$ since (3.1) holds and $c_{2}, d_{2}>0$. By Lemma 3.1, we have

$$
\lim _{t \rightarrow+\infty} z(t)=0 \quad \text { a.s. }
$$

if $G_{3}<\tilde{G}_{3}$ and $\varepsilon$ is sufficiently small. Combining (3.10) with equation (3.2) gives

$$
\frac{1}{t} \ln \frac{x(t)}{x(0)} \geq r_{1}-\frac{\alpha_{1}^{2}}{2}-a_{11}[x(t)]-\varepsilon+\frac{\alpha_{1} B_{1}(t)}{t}
$$

for sufficiently large $t$. It follows from Lemma 3.1 that

$$
[x]_{*} \geq\left(r_{1}-\alpha_{1}^{2} / 2\right) / a_{11}>0 \quad \text { a.s., }
$$


which implies that

$$
\limsup _{t \rightarrow \infty} \ln x(t) / t=0 \quad \text { a.s. }
$$

if (3.1) holds. Let $c_{1}=a_{21}$ and $d_{1}=a_{11}$ in (3.6). It follows that

$$
\begin{aligned}
a_{11} \frac{1}{t} \ln \frac{y(t)}{y(0)}= & -a_{21} \frac{1}{t} \ln \frac{x(t)}{x(0)}+\rho_{2}-\tilde{\rho}_{2}-\left(a_{11} a_{22}-a_{12} a_{21}\right)[y(t)] \\
& -\left(a_{13} a_{21}+a_{11} a_{23}\right)[z(t)]+\frac{a_{21} \alpha_{1} B_{1}(t)}{t}+\frac{a_{11} \alpha_{2} B_{2}(t)}{t} \\
\leq & \rho_{2}-\tilde{\rho}_{2}-\left(a_{11} a_{22}-a_{12} a_{21}\right)[y(t)]+\varepsilon+\frac{a_{21} \alpha_{1} B_{1}(t)}{t}+\frac{a_{11} \alpha_{2} B_{2}(t)}{t}
\end{aligned}
$$

for sufficiently large $t$ if (3.10), (3.11) and (3.1) hold. It follows from Lemma 3.1 that

$$
\lim _{t \rightarrow+\infty} y(t)=0 \quad \text { a.s. }
$$

if $\rho_{2}<\tilde{\rho}_{2}$ and $\varepsilon$ is sufficiently small. Combining equation (3.2) with (3.10) and (3.14) gives

$$
\frac{1}{t} \ln \frac{y(t)}{y(0)} \leq r_{1}-\frac{\alpha_{1}^{2}}{2}+\varepsilon-a_{11}[x(t)]+\frac{\alpha_{1} B_{1}(t)}{t}
$$

for sufficiently large $t$. By applying Lemma 3.1, we get

$$
[x]^{*} \leq\left(r_{1}-\alpha_{1}^{2} / 2\right) / a_{11} \quad \text { a.s. }
$$

Then we can combine (3.11) and (3.15) to obtain

$$
\lim _{t \rightarrow+\infty} \frac{1}{t} \int_{0}^{t} x(s) d s=\frac{r_{1}-\alpha_{1}^{2} / 2}{a_{11}} \text { a.s. }
$$

In view of (3.10), (3.14) and (3.16), (i) of Theorem 2.3 holds.

(ii) It follows from (3.10), (3.3), (3.1) and Lemma 3.1 that

$$
\frac{1}{t} \ln \frac{y(t)}{y(0)} \geq r_{2}-\frac{\alpha_{2}^{2}}{2}-a_{22}[y(t)]-\varepsilon+\frac{\alpha_{1} B_{2}(t)}{t}, \quad[y]_{*} \geq\left(r_{2}-\alpha_{2}^{2} / 2\right) / a_{22}>0 \quad \text { a.s. }
$$

for sufficiently large $t$ and

$$
\limsup _{t \rightarrow \infty} \ln y(t) / t=0 \quad \text { a.s. }
$$

Let $c_{1}=a_{22}$ and $d_{1}=a_{12}$ in (3.6). It follows that

$$
\begin{aligned}
a_{22} \frac{1}{t} \ln \frac{x(t)}{x(0)}= & -a_{12} \frac{1}{t} \ln \frac{y(t)}{y(0)}+\rho_{1}-\tilde{\rho}_{1}-\left(a_{11} a_{22}-a_{12} a_{21}\right)[x(t)] \\
& -\left(a_{13} a_{22}+a_{12} a_{23}\right)[z(t)]+\frac{a_{22} \alpha_{1} B_{1}(t)}{t}+\frac{a_{12} \alpha_{2} B_{2}(t)}{t}
\end{aligned}
$$




$$
\leq \rho_{1}-\tilde{\rho}_{1}+\varepsilon-\left(a_{11} a_{22}-a_{12} a_{21}\right)[x(t)]+\frac{a_{22} \alpha_{1} B_{1}(t)}{t}+\frac{a_{12} \alpha_{2} B_{2}(t)}{t}
$$

for sufficiently large $t$ if (3.1) and (3.18) hold. Then

$$
\lim _{t \rightarrow+\infty} x(t)=0 \quad \text { a.s. }
$$

if $\rho_{1}<\tilde{\rho}_{1}$ and $\varepsilon$ is sufficiently small. From (3.10), (3.20) and Lemma 3.1, we have

$$
\frac{1}{t} \ln \frac{y(t)}{y(0)} \leq r_{2}-\frac{\alpha_{2}^{2}}{2}+\varepsilon-a_{22}[y(t)]+\frac{\alpha_{2} B_{2}(t)}{t}
$$

for sufficiently large $t$ and

$$
[y]^{*} \leq\left(r_{2}-\alpha_{2}^{2} / 2\right) / a_{22} \quad \text { a.s. }
$$

if $r_{2}>\alpha_{2}^{2} / 2$ and $\varepsilon$ is sufficiently small. It follows from (3.10), (3.20), (3.17) and (3.21) that (ii) of Theorem 2.3 holds.

(iii) We first prove that

$$
\lim _{t \rightarrow+\infty} x(t)=0 \quad \text { a.s. and } \quad \lim _{t \rightarrow+\infty} y(t)=0 \text { a.s. }
$$

Let $c_{1}=a_{22}$ and $d_{1}=a_{12}$ in (3.6). From (3.19) and $\rho_{1}<\tilde{\rho}_{1}$, we conclude that (3.7) or (3.8) holds. If (3.7) holds, then equation (3.3) multiplied by $a_{33}$ minus equation (3.4) multiplied by $a_{23}$ gives

$$
\begin{aligned}
a_{33} \frac{1}{t} \ln \frac{y(t)}{y(0)}= & a_{23} \frac{1}{t} \ln \frac{z(t)}{z(0)}+\rho_{5}-\tilde{\rho}_{5}+\left(a_{21} a_{33}+a_{23} a_{31}\right)[x(t)] \\
& -\left(a_{22} a_{33}-a_{23} a_{32}\right)[y(t)]+\frac{a_{33} \alpha_{2} B_{2}(t)}{t}-\frac{a_{23} \alpha_{3} B_{3}(t)}{t} \\
\leq & \rho_{5}-\tilde{\rho}_{5}+\varepsilon-\left(a_{22} a_{33}-a_{23} a_{32}\right)[y(t)]+\frac{a_{33} \alpha_{2} B_{2}(t)}{t}-\frac{a_{23} \alpha_{3} B_{3}(t)}{t} .
\end{aligned}
$$

If follows from $\left(\mathrm{A}_{1}\right)$ and Lemma 3.1 that $\lim _{t \rightarrow+\infty} y(t)=0$ a.s. If (3.8) holds, then equation (3.2) multiplied by $a_{33}$ minus equation (3.4) multiplied by $a_{13}$ gives

$$
\begin{aligned}
a_{33} \frac{1}{t} \ln \frac{x(t)}{x(0)}= & a_{13} \frac{1}{t} \ln \frac{z(t)}{z(0)}+\rho_{3}-\tilde{\rho}_{3}-\left(a_{11} a_{33}-a_{13} a_{31}\right)[x(t)] \\
& +\left(a_{12} a_{33}+a_{13} a_{32}\right)[y(t)]+\frac{a_{33} \alpha_{1} B_{1}(t)}{t}-\frac{a_{13} \alpha_{3} B_{3}(t)}{t} \\
\leq & \rho_{3}-\tilde{\rho}_{3}+\varepsilon-\left(a_{11} a_{33}-a_{13} a_{31}\right)[x(t)]+\frac{a_{33} \alpha_{1} B_{1}(t)}{t}-\frac{a_{13} \alpha_{3} B_{3}(t)}{t} .
\end{aligned}
$$

From $\left(\mathrm{A}_{2}\right)$ and Lemma 3.1, we have $\lim _{t \rightarrow+\infty} x(t)=0$ a.s. Similarly, if $\left(\mathrm{A}_{3}\right)$ or $\left(\mathrm{A}_{4}\right)$ holds, then (3.22) holds.

It follows from (3.5) and Lemma 3.1 that

$$
[z]^{*} \leq\left(r_{3}-\alpha_{3}^{2} / 2\right) / a_{33} \quad \text { a.s. }
$$


if $r_{3}>\alpha_{3}^{2} / 2$. On the other hand, by applying (3.22) and Lemma 3.1, we have

$$
\frac{1}{t} \ln \frac{z(t)}{z(0)} \geq r_{3}-\frac{\alpha_{3}^{2}}{2}-\varepsilon-a_{33}[z(t)]+\frac{\alpha_{3} B_{3}(t)}{t}
$$

and

$$
[z]_{*} \geq\left(r_{3}-\alpha_{3}^{2} / 2\right) / a_{33} \quad \text { a.s. }
$$

It follows from (3.22), (3.25) and (3.26) that (iii) of Theorem 2.3 holds.

Proof of Theorem 2.4 We first establish (iv) of Theorem 2.4. It follows from (3.9) and Lemma 3.1 that $\lim _{t \rightarrow+\infty} z(t)=0$ a.s. From (3.13), we have

$$
a_{11} \frac{1}{t} \ln \frac{y(t)}{y(0)} \geq \rho_{2}-\tilde{\rho}_{2}-\varepsilon-\left(a_{11} a_{22}-a_{12} a_{21}\right)[y(t)]+\frac{a_{21} \alpha_{1} B_{1}(t)}{t}+\frac{a_{11} \alpha_{2} B_{2}(t)}{t}
$$

for sufficiently large $t$. By applying (6), Lemma 3.1 and the arbitrariness of $\varepsilon$, we obtain

$$
[y]_{*} \geq\left(\rho_{2}-\tilde{\rho}_{2}\right) /\left(a_{11} a_{22}-a_{12} a_{21}\right) \quad \text { a.s. }
$$

and

$$
\limsup _{t \rightarrow \infty} \ln y(t) / t=0 \quad \text { a.s. }
$$

Substituting (3.27) into (3.2) leads to

$$
\frac{1}{t} \ln \frac{x(t)}{x(0)} \geq r_{1}-\frac{\alpha_{1}^{2}}{2}-a_{11}[x(t)]+a_{12}[y]_{*}-a_{13}[z(t)]+\frac{\alpha_{1} B_{1}(t)}{t} .
$$

This implies that

$$
[x]_{*} \geq\left(\rho_{1}-\tilde{\rho}_{1}\right) /\left(a_{11} a_{22}-a_{12} a_{21}\right) \quad \text { a.s. }
$$

by Lemma 3.1 and $\varepsilon$ is sufficiently small. On the other hand, it follows from (3.19) that

$$
a_{22} \frac{1}{t} \ln \frac{x(t)}{x(0)} \leq \rho_{1}-\tilde{\rho}_{1}+\varepsilon-\left(a_{11} a_{22}-a_{12} a_{21}\right)[x(t)]+\frac{a_{22} \alpha_{1} B_{1}(t)}{t}+\frac{a_{12} \alpha_{2} B_{2}(t)}{t}
$$

for sufficiently large $t$ if (3.1) and (3.28) hold. It follows from Lemma 3.1, the arbitrariness of $\varepsilon$ and $\rho_{1}>\tilde{\rho}_{1}$ that

$$
[x]^{*} \leq\left(\rho_{1}-\tilde{\rho}_{1}\right) /\left(a_{11} a_{22}-a_{12} a_{21}\right) \quad \text { a.s. }
$$

Similarly, from (3.13) and $\rho_{2}>\tilde{\rho}_{2}$, we get

$$
[y]^{*} \leq\left(\rho_{2}-\tilde{\rho}_{2}\right) /\left(a_{11} a_{22}-a_{12} a_{21}\right) \quad \text { a.s. }
$$

It follows from (3.27), (3.29), (3.30) and (3.31) that (iv) of Theorem 2.4 holds. 
We now establish (v) of Theorem 2.4. From (3.9) and Lemma 3.1, we conclude that

$$
[z]^{*} \leq\left(G_{3}-\tilde{G}_{3}\right) / G
$$

It follows from (3.19) and (3.32) that

$$
\begin{aligned}
a_{22} \frac{1}{t} \ln \frac{x(t)}{x(0)} \geq & \rho_{1}-\tilde{\rho}_{1}-\varepsilon-\left(a_{11} a_{22}-a_{12} a_{21}\right)[x(t)] \\
& -\left(a_{13} a_{22}+a_{12} a_{23}\right)[z]^{*}+\frac{a_{22} \alpha_{1} B_{1}(t)}{t}+\frac{a_{12} \alpha_{2} B_{2}(t)}{t} \\
\geq & \left(a_{11} a_{22}-a_{12} a_{21}\right)\left(G_{1}-\tilde{G}_{1}\right) / G-\varepsilon \\
& -\left(a_{11} a_{22}-a_{12} a_{21}\right)[x(t)]+\frac{a_{22} \alpha_{1} B_{1}(t)}{t}+\frac{a_{12} \alpha_{2} B_{2}(t)}{t}
\end{aligned}
$$

for sufficiently large $t$. By applying Lemma 3.1, one obtains

$$
[x]_{*} \geq\left(G_{1}-\tilde{G}_{1}\right) / G>0 \text { a.s. }
$$

for $G_{1}>\tilde{G}_{1}$, and $\varepsilon$ is sufficiently small. Let

$$
\begin{aligned}
& c_{3}=-\left(a_{21} a_{33}+a_{23} a_{31}\right) /\left(a_{11} a_{33}-a_{13} a_{31}\right), \\
& d_{3}=\left(a_{11} a_{23}+a_{13} a_{21}\right) /\left(a_{11} a_{33}-a_{13} a_{31}\right) .
\end{aligned}
$$

Multiplying both sides of (3.2), (3.3) and (3.4) by $c_{3},-1$ and $d_{3}$, respectively, and then adding these three equalities yield

$$
\begin{aligned}
\frac{1}{t} \ln \frac{y(t)}{y(0)}= & \frac{c_{3}}{t} \ln \frac{x(t)}{x(0)}+\frac{d_{3}}{t} \ln \frac{z(t)}{z(0)}+\frac{G_{2}-\tilde{G}_{2}}{a_{11} a_{33}-a_{13} a_{31}}-\frac{G}{a_{11} a_{33}-a_{13} a_{31}}[y(t)] \\
& +\frac{\alpha_{2} B_{2}(t)-\alpha_{1} c_{3} B_{1}(t)-\alpha_{3} d_{3} B_{3}(t)}{t} \\
\leq & \frac{G_{2}-\tilde{G}_{2}}{a_{11} a_{33}-a_{13} a_{31}}+\varepsilon-\frac{G}{a_{11} a_{33}-a_{13} a_{31}}[y(t)] \\
& +\frac{\alpha_{2} B_{2}(t)-\alpha_{1} c_{3} B_{1}(t)-\alpha_{3} d_{3} B_{3}(t)}{t}
\end{aligned}
$$

for sufficiently large $t$ if (3.34) and (3.1) hold. It follows from Lemma 3.1 that

$$
\lim _{t \rightarrow+\infty} y(t)=0 \quad \text { a.s. }
$$

if $G_{2}<\tilde{G}_{2}$ and $\varepsilon$ is sufficiently small. Next, using (3.24), Lemma 3.1 and $\rho_{3}>\tilde{\rho}_{3}$, one gets

$$
[x]^{*} \leq\left(\rho_{3}-\tilde{\rho}_{3}\right) /\left(a_{11} a_{33}-a_{13} a_{31}\right) \quad \text { a.s. }
$$

Equation (3.4) multiplied by $a_{11}$ minus equation (3.2) multiplied by $a_{31}$ gives

$$
a_{11} \frac{1}{t} \ln \frac{z(t)}{z(0)} \leq \rho_{4}-\tilde{\rho}_{4}+\varepsilon-\left(a_{11} a_{33}-a_{13} a_{31}\right)[z(t)]+\frac{a_{11} \alpha_{3} B_{3}(t)}{t}-\frac{a_{31} \alpha_{1} B_{1}(t)}{t} .
$$


It follows from Lemma 3.1 that

$$
[z]^{*} \leq\left(\rho_{4}-\tilde{\rho}_{4}\right) /\left(a_{11} a_{33}-a_{13} a_{31}\right) \quad \text { a.s. }
$$

for $\rho_{4}>\tilde{\rho}_{4}$. On the other hand, substituting (3.38) and (3.36) into (3.2) yields

$$
\begin{aligned}
\frac{1}{t} \ln \frac{x(t)}{x(0)} & \geq r_{1}-\frac{\alpha_{1}^{2}}{2}-\varepsilon-a_{11}[x(t)]-a_{13}[z]^{*}+\frac{\alpha_{1} B_{1}(t)}{t} \\
& \geq \frac{a_{11}\left(\rho_{3}-\tilde{\rho}_{3}\right)}{a_{11} a_{33}-a_{13} a_{31}}-\varepsilon-a_{11}[x(t)]+\frac{\alpha_{1} B_{1}(t)}{t}
\end{aligned}
$$

for sufficiently large $t$. Then

$$
[x]_{*} \geq\left(\rho_{3}-\tilde{\rho}_{3}\right) /\left(a_{11} a_{33}-a_{13} a_{31}\right) \quad \text { a.s. }
$$

if $\varepsilon$ is sufficiently small and $\rho_{3}>\tilde{\rho}_{3}$. Applying Lemma 3.1 and substituting (3.39) and (3.36) into (3.4) yield

$$
\begin{aligned}
\frac{1}{t} \ln \frac{z(t)}{z(0)} & \geq r_{3}-\frac{\alpha_{3}^{2}}{2}-a_{31}[x]^{*}-\varepsilon-a_{33}[z(t)]+\frac{\alpha_{3} B_{3}(t)}{t} \\
& \geq \frac{a_{33}\left(\rho_{4}-\tilde{\rho}_{4}\right)}{a_{11} a_{33}-a_{13} a_{31}}-\varepsilon-a_{33}[z(t)]+\frac{\alpha_{3} B_{3}(t)}{t}
\end{aligned}
$$

and

$$
[z]_{*} \geq\left(\rho_{4}-\tilde{\rho}_{4}\right) /\left(a_{11} a_{33}-a_{13} a_{31}\right) \quad \text { a.s. }
$$

It follows from (3.36)-(3.40) that (v) of Theorem 2.4 holds.

We finally establish (vi) of Theorem 2.4. Substituting (3.32) into (3.13) and applying Lemma 3.1 yield

$$
\begin{aligned}
a_{11} \frac{1}{t} \ln \frac{y(t)}{y(0)} \geq & \rho_{2}-\tilde{\rho}_{2}-\varepsilon-\left(a_{11} a_{22}-a_{12} a_{21}\right)[y(t)] \\
& -\left(a_{13} a_{21}+a_{11} a_{23}\right)[z]^{*}+\frac{a_{21} \alpha_{1} B_{1}(t)}{t}+\frac{a_{11} \alpha_{2} B_{2}(t)}{t} \\
\geq & \left(a_{11} a_{22}-a_{12} a_{21}\right)\left(G_{2}-\tilde{G}_{2}\right) / G-\varepsilon \\
& -\left(a_{11} a_{22}-a_{12} a_{21}\right)[y(t)]+\frac{a_{21} \alpha_{1} B_{1}(t)}{t}+\frac{a_{11} \alpha_{2} B_{2}(t)}{t}
\end{aligned}
$$

for sufficiently large $t$ and

$$
[y]_{*} \geq\left(G_{2}-\tilde{G}_{2}\right) / G>0 \text { a.s. }
$$

for $G_{2}>\tilde{G}_{2}$, and $\varepsilon$ is sufficiently small. Let

$$
c_{4}=-\left(a_{12} a_{33}+a_{13} a_{32}\right) /\left(a_{22} a_{33}-a_{23} a_{32}\right), \quad d_{4}=\left(a_{12} a_{23}+a_{13} a_{22}\right) /\left(a_{22} a_{33}-a_{23} a_{32}\right) .
$$


Multiplying both sides of (3.2), (3.3) and (3.4) by $-1, c_{4}$ and $d_{4}$, respectively, and adding these three equalities, we have

$$
\begin{aligned}
\frac{1}{t} \ln \frac{x(t)}{x(0)}= & \frac{c_{4}}{t} \ln \frac{y(t)}{y(0)}+\frac{d_{4}}{t} \ln \frac{z(t)}{z(0)} \\
& +\frac{G_{1}-\tilde{G}_{1}}{a_{22} a_{33}-a_{23} a_{32}}-\frac{G}{a_{22} a_{33}-a_{23} a_{32}}[x(t)] \\
& +\frac{\alpha_{1} B_{1}(t)-\alpha_{2} c_{4} B_{2}(t)-\alpha_{3} d_{4} B_{3}(t)}{t} \\
\leq & \frac{G_{1}-\tilde{G}_{1}}{a_{22} a_{33}-a_{23} a_{32}}+\varepsilon-\frac{G}{a_{22} a_{33}-a_{23} a_{32}}[x(t)] \\
& +\frac{\alpha_{1} B_{1}(t)-\alpha_{2} c_{4} B_{2}(t)-\alpha_{3} d_{4} B_{3}(t)}{t}
\end{aligned}
$$

for sufficiently large $t$ since (3.42) and (3.1) hold. Then

$$
\lim _{t \rightarrow+\infty} x(t)=0 \quad \text { a.s. }
$$

if $G_{1}<\tilde{G}_{1}, a_{22} a_{33}>a_{23} a_{32}$ and $\varepsilon$ is sufficiently small. From (3.23), Lemma 3.1 and $\rho_{5}>\tilde{\rho}_{5}$, one has

$$
[y]^{*} \leq\left(\rho_{5}-\tilde{\rho}_{5}\right) /\left(a_{22} a_{33}-a_{23} a_{32}\right) \quad \text { a.s. }
$$

Using (3.44), (3.3), (3.4) and similar arguments as the ones given by (v) of Theorem 2.4, we derive

$$
\begin{aligned}
& {[y]_{*} \geq\left(\rho_{5}-\tilde{\rho}_{5}\right) /\left(a_{22} a_{33}-a_{23} a_{32}\right) \quad \text { a.s., }} \\
& {[z]_{*} \geq\left(\rho_{6}-\tilde{\rho}_{6}\right) /\left(a_{22} a_{33}-a_{23} a_{32}\right) \quad \text { a.s., }} \\
& {[z]^{*} \leq\left(\rho_{6}-\tilde{\rho}_{6}\right) /\left(a_{22} a_{33}-a_{23} a_{32}\right) \quad \text { a.s. }}
\end{aligned}
$$

This yields (vi) of Theorem 2.4.

Proof of Theorem 2.5 From the above discussion, we conclude that if $a_{11} a_{22}>a_{12} a_{21}$ and $G_{i}>\tilde{G}_{i}, i=1,2,3$, hold, then we have (3.32), (3.34) and (3.42). Meanwhile, it follows from (3.9) and Lemma 3.1 that

$$
\begin{aligned}
\frac{1}{t} \ln \frac{z(t)}{z(0)} \geq & \frac{G_{3}-\tilde{G}_{3}}{a_{11} a_{22}-a_{12} a_{21}}-\varepsilon-\frac{G}{a_{11} a_{22}-a_{12} a_{21}}[z(t)] \\
& +\frac{\alpha_{3} B_{3}(t)-\alpha_{1} c_{2} B_{1}(t)-\alpha_{2} d_{2} B_{2}(t)}{t}
\end{aligned}
$$

for sufficiently large $t$, and

$$
[z]_{*} \geq\left(G_{3}-\tilde{G}_{3}\right) / G \text { a.s. }
$$


By (3.35) and (3.43), we have

$$
\begin{aligned}
\frac{1}{t} \ln \frac{y(t)}{y(0)} \leq & \frac{G_{2}-\tilde{G}_{2}}{a_{11} a_{33}-a_{13} a_{31}}+\varepsilon-\frac{G}{a_{11} a_{33}-a_{13} a_{31}}[y(t)] \\
& +\frac{\alpha_{2} B_{2}(t)-\alpha_{1} c_{3} B_{1}(t)-\alpha_{3} d_{3} B_{3}(t)}{t}
\end{aligned}
$$

and

$$
\begin{aligned}
\frac{1}{t} \ln \frac{x(t)}{x(0)} \leq & \frac{G_{1}-\tilde{G}_{1}}{a_{22} a_{33}-a_{23} a_{32}}+\varepsilon-\frac{G}{a_{22} a_{33}-a_{23} a_{32}}[x(t)] \\
& +\frac{\alpha_{1} B_{1}(t)-\alpha_{2} c_{4} B_{2}(t)-\alpha_{3} d_{4} B_{3}(t)}{t}
\end{aligned}
$$

for sufficiently large $t$. Then

$$
[x]^{*} \leq\left(G_{1}-\tilde{G}_{1}\right) / G \text { a.s. and }[y]^{*} \leq\left(G_{2}-\tilde{G}_{2}\right) / G \text { a.s. }
$$

From (3.32), (3.34), (3.42), (3.47) and (3.48), we conclude that Theorem 2.5 holds. This completes the proof of Theorem 2.5 .

\subsection{Proof of Theorem 2.6}

To prove Theorem 2.6, we need the following lemmas.

Lemma 3.3 (see [40]) If $g$ is a non-negative function defined on $[0,+\infty)$ such that $g$ is integrable and uniformly continuous, then $\lim _{t \rightarrow+\infty} g(t)=0$.

Let $(\Omega, F, P)$ be a probability space, and let $(E, B)$ be a measurable space. A family of random variables $\left\{X_{t}\right\}_{t \in T}$ satisfies $X_{t}:(\Omega, F) \rightarrow(E, B)$ for $t \in T$ and for all $\omega \in \Omega$. The mapping $X_{t}(\cdot, \omega): t \in T \rightarrow X_{t}(\omega) \in E$ is called a sample path of the process corresponding to $\omega$.

Carrying out similar arguments as those of Lemma 15 in [25], we have the following lemma.

Lemma 3.4 Let $(x(t), y(t), z(t))^{T}$ be a positive solution of (1.2). Then almost every sample path of $x(t), y(t)$ and $z(t)$ is uniformly continuous.

Proof of Theorem 2.6 Let $\left(x_{i}(t), y_{i}(t), z_{i}(t)\right)^{T}, i=1,2$, be two arbitrary solutions of model (1.2) with the initial values $\left(x_{i}(0), y_{i}(0), z_{i}(0)\right) \in \mathbb{R}_{+}^{3}, i=1,2,3, \omega_{i}>0, i=1,2,3$, and

$$
V(t)=\lambda_{1}\left|\ln \left(x_{1}(t) / x_{2}(t)\right)\right|+\lambda_{2}\left|\ln \left(y_{1}(t) / y_{2}(t)\right)\right|+\lambda_{3}\left|\ln \left(z_{1}(t) / z_{2}(t)\right)\right|
$$

A direct calculation has

$$
\begin{aligned}
d V(t)= & \lambda_{1} \operatorname{sgn}\left(x_{1}(t)-x_{2}(t)\right)\left[-a_{11}\left(x_{1}(t)-x_{2}(t)\right)\right. \\
& \left.+a_{12}\left(y_{1}(t)-y_{2}(t)\right)-a_{13}\left(z_{1}(t)-z_{2}(t)\right)\right] d t \\
& +\lambda_{2} \operatorname{sgn}\left(y_{1}(t)-y_{2}(t)\right)\left[a_{21}\left(x_{1}(t)-x_{2}(t)\right)\right. \\
& \left.-a_{22}\left(y_{1}(t)-y_{2}(t)\right)-a_{23}\left(z_{1}(t)-z_{2}(t)\right)\right] d t
\end{aligned}
$$




$$
\begin{aligned}
& -\lambda_{3} \operatorname{sgn}\left(z_{1}(t)-z_{2}(t)\right)\left[a_{31}\left(x_{1}(t)-x_{2}(t)\right)\right. \\
& \left.+a_{32}\left(y_{1}(t)-y_{2}(t)\right)-a_{33}\left(z_{1}(t)-z_{2}(t)\right)\right] d t \\
\leq & -\left(\lambda_{1} a_{11}-\lambda_{2} a_{21}-\lambda_{3} a_{31}\right)\left|x_{1}(t)-x_{2}(t)\right| d t \\
& -\left(\lambda_{2} a_{22}-\lambda_{1} a_{12}-\lambda_{3} a_{32}\right)\left|y_{1}(t)-y_{2}(t)\right| d t \\
& -\left(\lambda_{3} a_{33}-\lambda_{1} a_{13}-\lambda_{2} a_{23}\right)\left|z_{1}(t)-z_{2}(t)\right| d t \\
:= & -R(t) d t .
\end{aligned}
$$

Then

$$
V(t)+\int_{0}^{t} R(s) d s \leq V(0)<+\infty
$$

Applying $V(t) \geq 0$, Lemma 3.3 and Lemma 3.4 shows that (1.2) is globally asymptotically stable.

\subsection{The proof of Theorem 2.7}

To complete the proof of Theorem 2.7, we first introduce the theory of Has'minskii [41]. Let $E^{l}$ be an $l$-dimensional Euclidean space and $Y(t)$ be a homogeneous Markov process in $E^{l}$. Moreover, $Y(t)$ satisfies the stochastic differential equation

$$
d Y(t)=b(Y) d t+\sum_{m=1}^{k} g_{m}(Y) d B_{m}(t) .
$$

Let $\Lambda(x)=\left(a_{i j}(x)\right)$ be the diffusion matrix, where $a_{i j}(x)=\sum_{m=1}^{k} g_{m}^{i}(x) g_{m}^{j}(x)$.

We are now presenting a useful condition. There is a bounded domain $U \subset E^{l}$ with the regular boundary $\Gamma$ such that

$\left(\mathrm{A}_{1}\right)$ In the domain $U$ and some neighborhood thereof, the smallest eigenvalue of the diffusion matrix $\Lambda(x)$ is bounded away from zero;

$\left(\mathrm{A}_{2}\right)$ If $x \in E^{l} \backslash U$, the mean time $\tau$ at which a path issuing from $x$ reaches the set $U$ is finite, and $\sup _{x \in K} E_{x} \tau<+\infty$ for every compact subset $K \subset E^{l}$.

In order to verify $\left(\mathrm{A}_{1}\right)$ and $\left(\mathrm{A}_{2}\right)$ in Assumption 3.1, we introduce two stronger conditions (see $[36,42,43])$ :

$\left(\mathrm{B}_{1}\right)$ To establish $\left(\mathrm{A}_{1}\right)$, it is sufficient to show that $T$ is uniformly elliptical in $U$, where $T u=b(x) u_{x}+\operatorname{tr}\left(\Lambda(x) u_{x x}\right) / 2$, that is, there exists $c>0$ such that $\sum_{i, j=1}^{k} a_{i j}(x) \xi_{i} \xi_{j} \geq$ $c|\xi|^{2}, x \in U, \xi \in R^{l}$

$\left(\mathrm{B}_{2}\right)$ To establish $\left(\mathrm{A}_{2}\right)$, it is sufficient to prove that there exist a neighborhood $U$ and a nonnegative $C^{2}$-function $V(x)$ such that, for any $x \in E^{l} \backslash U, L V(x)<0$.

Lemma 3.5 ([41]) If Assumption 3.1 holds, then the Markov process $Y(t)$ has a stationary distribution $\mu(\cdot)$. Furthermore, if $f(\cdot)$ is a function integrable with respect to the measure $\mu$, then

$$
P\left\{\lim _{t \rightarrow+\infty} \frac{1}{t} \int_{0}^{t} f(Y(s)) d s=\int_{E^{l}} f(x) \mu(d x)\right\}=1
$$


Proof of Theorem 2.7 In order to establish our result, we only need to verify that conditions $\left(\mathrm{B}_{1}\right)$ and $\left(\mathrm{B}_{2}\right)$ hold. Note that the diffusion matrix of (1.2) is $\Lambda(x)=\operatorname{diag}\left(\alpha_{1}^{2} x^{2}, \alpha_{2}^{2} y^{2}, \alpha_{3}^{2} z^{2}\right)$.

By $\delta_{i}>0, i=1,2,3$, and formula (2.2), we obtain that the ellipsoid

$$
\delta_{1}\left(x-x^{*}\right)^{2}+\delta_{2}\left(y-y^{*}\right)^{2}+\delta_{3}\left(z-z^{*}\right)^{2}=\alpha_{1}^{2} x^{*} / 2+\alpha_{2}^{2} y^{*} / 2+\alpha_{3}^{2} z^{*} / 2
$$

lies entirely in $\mathbb{R}_{+}^{3}$. A direct computation shows that there exists a positive constant $\gamma$ such that

$$
\sum_{i, j=1}^{3} a_{i j}(x) \omega_{i} \omega_{j}=\alpha_{1}^{2} x^{2} \omega_{1}^{2}+\alpha_{2}^{2} y^{2} \omega_{2}^{2}+\alpha_{3}^{2} z^{2} \omega_{3}^{2}>\gamma|\omega|^{2}
$$

for $x \in \bar{U}$ and $\omega \in \mathbb{R}^{3}$, where $U$ is a neighborhood of the ellipsoid with $\bar{U} \subseteq \mathbb{R}_{+}^{3}$. This shows that condition $\left(B_{1}\right)$ holds. On the other hand, we define

$$
V(x, y, z)=x-x^{*}-x^{*} \ln \left(x / x^{*}\right)+y-y^{*}-y^{*} \ln \left(y / y^{*}\right)+z-z^{*}-z^{*} \ln \left(z / z^{*}\right) .
$$

A simple calculation has

$$
d V(x, y, z)=L V(x, y, z) d t+\left(x-x^{*}\right) \alpha_{1} d B_{1}(t)+\left(y-y^{*}\right) \alpha_{2} d B_{2}(t)+\left(z-z^{*}\right) \alpha_{3} d B_{3}(t),
$$

where

$$
\begin{aligned}
L V(x, y, z)= & \left(x-x^{*}\right)\left[r_{1}-a_{11} x+a_{12} y-a_{13} z\right]+\alpha_{1}^{2} x^{*} / 2 \\
& +\left(y-y^{*}\right)\left[r_{2}+a_{21} x-a_{22} y-a_{23} z\right]+\alpha_{2}^{2} y^{*} / 2 \\
& +\left(z-z^{*}\right)\left[r_{3}-a_{31} x-a_{32} y-a_{33} z\right]+\alpha_{3}^{2} z^{*} / 2 \\
\leq & -\delta_{1}\left(x-x^{*}\right)^{2}-\delta_{2}\left(y-y^{*}\right)^{2}-\delta_{3}\left(z-z^{*}\right)^{2}+\left(\alpha_{1}^{2} x^{*}+\alpha_{2}^{2} y^{*}+\alpha_{3}^{2} z^{*}\right) / 2
\end{aligned}
$$

since $\left(x^{*}, y^{*}, z^{*}\right)^{T}$ is the positive equilibrium point of the corresponding deterministic model of (1.2). This implies that $L V(x)<0$ for any $x \in \mathbb{R}_{+}^{3} \backslash U$ and condition $\left(\mathrm{B}_{2}\right)$ holds. In view of the above arguments and Lemma 3.5 , we conclude that there is a stationary distribution $\mu(\cdot)$ for model (1.2) and it has the ergodic property.

Finally, we claim that (5) holds. Applying the dominated convergence theorem and Lemma 3.2 gives

$$
\begin{aligned}
& E\left[\lim _{t \rightarrow+\infty} \frac{1}{t} \int_{0}^{t}[x(s) \wedge Q] d s\right]=\lim _{t \rightarrow+\infty} \frac{1}{t} \int_{0}^{t} E[x(s) \wedge Q] d s \leq K, \\
& E\left[\lim _{t \rightarrow+\infty} \frac{1}{t} \int_{0}^{t}[y(s) \wedge Q] d s\right]=\lim _{t \rightarrow+\infty} \frac{1}{t} \int_{0}^{t} E[y(s) \wedge Q] d s \leq K, \\
& E\left[\lim _{t \rightarrow+\infty} \frac{1}{t} \int_{0}^{t}[z(s) \wedge Q] d s\right]=\lim _{t \rightarrow+\infty} \frac{1}{t} \int_{0}^{t} E[z(s) \wedge Q] d s \leq K
\end{aligned}
$$

for any $Q>0$, where $\wedge$ is minimal. It follows from the ergodic property that

$$
\int_{\mathbb{R}_{+}^{3}}\left[\omega_{1} \wedge Q\right] \mu\left(d \omega_{1}, d \omega_{2}, d \omega_{3}\right)=E \lim _{t \rightarrow+\infty} \frac{1}{t} \int_{0}^{t}[x(s) \wedge Q] d s<K,
$$




$$
\begin{aligned}
& \int_{\mathbb{R}_{+}^{3}}\left[\omega_{2} \wedge Q\right] \mu\left(d \omega_{1}, d \omega_{2}, d \omega_{3}\right)=E \lim _{t \rightarrow+\infty} \frac{1}{t} \int_{0}^{t}[y(s) \wedge Q] d s<K, \\
& \int_{\mathbb{R}_{+}^{3}}\left[\omega_{3} \wedge Q\right] \mu\left(d \omega_{1}, d \omega_{2}, d \omega_{3}\right)=E \lim _{t \rightarrow+\infty} \frac{1}{t} \int_{0}^{t}[z(s) \wedge Q] d s<K .
\end{aligned}
$$

Letting $Q \rightarrow+\infty$ yields

$$
\begin{aligned}
& \int_{\mathbb{R}_{+}^{3}} \omega_{1} \mu\left(d \omega_{1}, d \omega_{2}, d \omega_{3}\right) \leq K, \\
& \int_{\mathbb{R}_{+}^{3}} \omega_{2} \mu\left(d \omega_{1}, d \omega_{2}, d \omega_{3}\right) \leq K, \\
& \int_{\mathbb{R}_{+}^{3}} \omega_{3} \mu\left(d \omega_{1}, d \omega_{2}, d \omega_{3}\right) \leq K .
\end{aligned}
$$

As a consequence of Lemma 3.5, (2.3) holds. This completes the proof of the theorem.

\section{Numerical simulations}

In this section, we do some numerical simulations to illustrate or complement our mathematical findings by using Milstein's method [44]. By taking advantage of revising the stochastic increment, Milstein's method achieves the high order convergence and can be more accurate to approximate the exact solution.

Let

$$
\begin{array}{lll}
r_{1}=0.5, & r_{2}=0.25, & r_{3}=0.4, \\
a_{11}=0.8, & a_{12}=0.2, & a_{13}=0.2, \\
a_{21}=0.3, & a_{22}=0.9, & a_{23}=0.1, \\
a_{31}=0.3, & a_{32}=0.1, & a_{33}=0.5 .
\end{array}
$$

A simple calculation gives

$$
\begin{array}{llll}
G=0.256, & G_{1}=0.17, & G_{2}=0.119, & G_{3}=0.079, \\
a_{11}>a_{12}, & a_{22}>a_{21}, & a_{11} a_{33}>a_{13} a_{31}, & a_{22} a_{33}>a_{23} a_{32}, \\
\rho_{1}=0.5, & \rho_{2}=0.35, & \rho_{3}=0.17, \\
\rho_{4}=0.17, & \rho_{5}=0.085, & \rho_{6}=0.335 .
\end{array}
$$

We choose the appropriate parameters $\alpha_{i}, i=1,2,3$, satisfying the conditions of our main theorems.

Figure 1(a) means that if the white noise is sufficiently large, then it is harmful to all the species and leads to the extinction of the species. In Figure 1(b), (c) and (d), we conclude that in the appropriate conditions, one species is stochastically persistent, and the other two species are extinct a.s.

Figure 2 shows that in the complex ecological relationship, the noise may have a positive effect on the coexistence of species, such as $\alpha_{1}, \alpha_{2}$ on $z$ and $\alpha_{3}$ on $x, y$. Therefore, in a certain context, noise may be beneficial to the maintenance of biodiversity. 
(a)

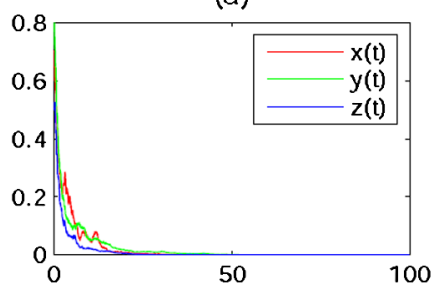

(c)

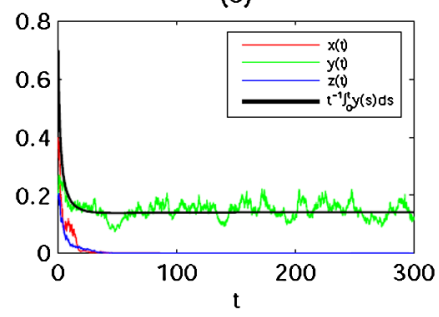

(b)

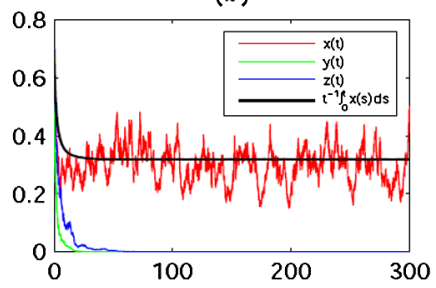

(d)

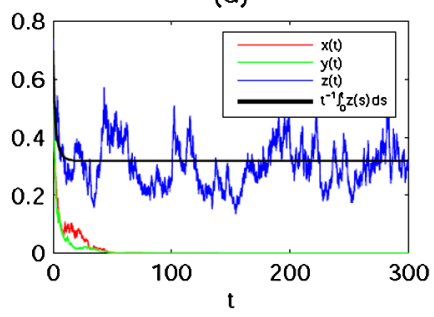

Figure 1 (a) Let $\alpha_{1}=1.1, \alpha_{2}=0.8$ and $\alpha_{3}=1$ such that $r_{i}<\alpha_{i}^{2} / 2, i=1,2,3$. It follows from Theorem 2.2 that all the species of model (1.2) go to extinction a.s. (b) Let $\alpha_{1}=0.7, \alpha_{2}=1$ and $\alpha_{3}=0.85$ such that $r_{1}>\alpha_{1}^{2} / 2$, $\rho_{2}<\tilde{\rho}_{2}$, and $G_{3}<\tilde{G}_{3}((i)$ of Theorem 2.3). Then the species $y(t)$ and $z(t)$ go to extinction a.s. and $x(t)$ is stochastically persistent with $\lim _{t \rightarrow+\infty} \frac{1}{t} \int_{0}^{t} x(s) d s=\frac{r_{1}-\alpha_{1}^{2} / 2}{a_{11}}=0.31875$. (c) Let $\alpha_{1}=1.1, \alpha_{2}=0.5$ and $\alpha_{3}=1$ such that $r_{2}>\alpha_{2}^{2} / 2, \rho_{1}<\tilde{\rho}_{1}$ and $G_{3}<\tilde{G}_{3}$ ((ii) of Theorem 2.3). Then the species $x(t)$ and $z(t)$ go to extinction a.s. and $y(t)$ is stochastically persistent with $\lim _{t \rightarrow+\infty} \frac{1}{t} \int_{0}^{t} y(s) d s=\frac{r_{2}-\alpha_{2}^{2} / 2}{a_{22}}=0.1389$. (d) Let $\alpha_{1}=1, \alpha_{2}=0.8$ and $\alpha_{3}=0.7$ such that $r_{3}>\alpha_{3}^{2} / 2, \rho_{1}<\tilde{\rho}_{1}$ and $\rho_{5}<\tilde{\rho}_{5}$ ((iii) of Theorem 2.3). Then the species $x(t)$ and $y(t)$ go to extinction a.s. and $z(t)$ is stochastically persistent with $\lim _{t \rightarrow+\infty} \frac{1}{t} \int_{0}^{t} z(s) d s=\frac{r_{3}-\alpha_{3}^{2} / 2}{a_{33}}=0.31$.

Figure 4 shows that if a stationary distribution has the ergodic property, then the mean of population density in time with the development of time is equal to the mean of population density in space.

\section{Conclusions}

Our results provide insight into dynamic properties of a stochastic cooperationcompetition model including stochastic persistence and stochastic extinction of species, global asymptotic stability of positive solutions and the existence of stationary distribution. The theoretical analysis shows that there are eight different cases for stochastic persistence and stochastic extinction of species. Especially, we also establish a sufficient criterion for global asymptotic stability of the positive solutions of model (1.2). A stationary distribution of model (1.2) with the ergodic property is investigated. This study shows that the time average of population size with the development of time is equal to the stationary distribution in space.

It is important and interesting to explore the effect of the noise on the persistence or extinction of species. To do this, we need to investigate the conclusions of Theorems 2.22.5 and evaluate the impact of the white noise intensity $\alpha_{i}(i=1,2,3)$ on these conclusions. The summary of the effect of the noise on species is listed in Table 1. Based on the analysis above, we may draw two conclusions:

- the noise is a harmful factor for single species in general, such as $\alpha_{1}$ for $x, \alpha_{2}$ for $y$ and $\alpha_{3}$ for $z$;

- in the complex ecological relationship, the noise may also have a positive effect on the coexistence of species, such as $\alpha_{1}, \alpha_{2}$ on $z$ and $\alpha_{3}$ on $x, y$. 
(a)

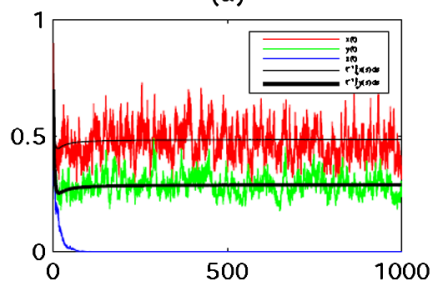

(c)

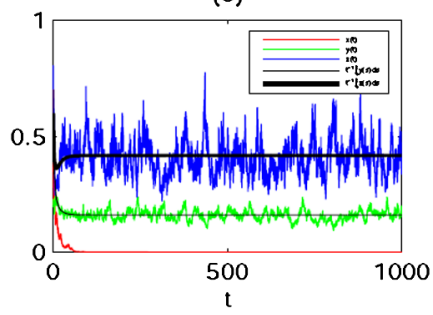

(b)

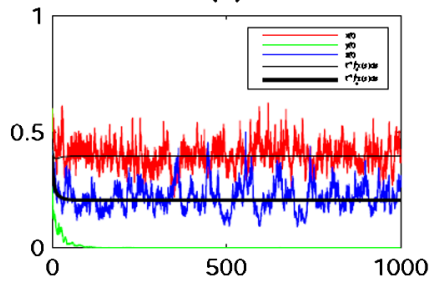

(d)

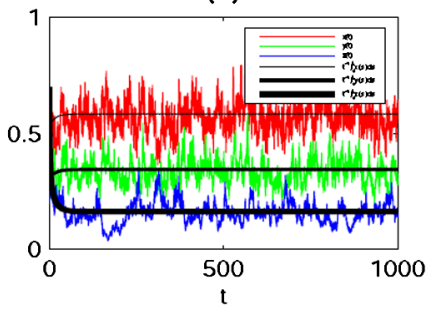

Figure 2 (a) $\alpha_{1}=0.6, \alpha_{2}=0.5$ and $\alpha_{3}=0.74$ such that $\rho_{1}>\tilde{\rho}_{1}, \rho_{2}>\tilde{\rho}_{2}$ and $G_{3}<\tilde{G}_{3}$ ((iv) of Theorem 2.4). Then the species $z(t)$ goes to extinction a.s. and the species $x(t)$ and $y(t)$ are stochastically persistent with $\lim _{t \rightarrow+\infty} \frac{1}{t} \int_{0}^{t} x(s) d s=\frac{\rho_{1}-\tilde{\rho}_{1}}{a_{11} a_{22}-a_{12} a_{21}}=0.4742, \lim _{t \rightarrow+\infty} \frac{1}{t} \int_{0}^{t} y(s) d s=\frac{\rho_{2}-\tilde{\rho}_{2}}{a_{11} a_{22}-a_{12} a_{21}}=0.2970$. (b) Let $\alpha_{1}=0.53$, $\alpha_{2}=0.84$ and $\alpha_{3}=0.6$ such that $\rho_{3}>\tilde{\rho}_{3}, \rho_{4}>\tilde{\rho}_{4}$ and $G_{2}<\tilde{G}_{2}((v)$ of Theorem 2.4). Then the species $y(t)$ goes to extinction a.s. and the species $x(t)$ and $z(t)$ are stochastically persistent with $\lim _{t \rightarrow+\infty} \frac{1}{t} \int_{0}^{t} x(s) d s=\frac{\rho_{3}-\tilde{\rho}_{3}}{a_{11} a_{33}-a_{13} a_{31}}=0.3993, \lim _{t \rightarrow+\infty} \frac{1}{t} \int_{0}^{t} z(s) d s=\frac{\rho_{4}-\tilde{\rho}_{4}}{a_{11} a_{33}-a_{13} a_{31}}=0.2003$. (c) Let $\alpha_{1}=0.98$, $\alpha_{2}=0.36$ and $\alpha_{3}=0.6$ such that $\rho_{5}>\tilde{\rho}_{5}, \rho_{6}>\tilde{\rho}_{6}$ and $G_{1}>\tilde{G}_{1}$ ((vi) of Theorem 2.4). Then the species $x(t)$ goes to extinction a.s. and the species $y(t)$ and $z(t)$ are stochastically persistent with

$\lim _{t \rightarrow+\infty} \frac{1}{t} \int_{0}^{t} y(s) d s=\frac{\rho_{5}-\tilde{\rho}_{5}}{a_{22} a_{33}-a_{23} a_{32}}=0.1605, \lim _{t \rightarrow+\infty} \frac{1}{t} \int_{0}^{t} z(s) d s=\frac{\rho_{6}-\tilde{\rho}_{6}}{a_{22} a_{33}-a_{23} a_{32}}=0.4079$. (d) Let $\alpha_{1}=0.4$, $\alpha_{2}=0.45$ and $\alpha_{3}=0.48$ such that $G_{1}>\tilde{G}_{1}, G_{2}>\tilde{G}_{2}$ and $G_{3}>\tilde{G}_{3}$ (Theorem 2.5). Then all the species are stochastically persistent with $\lim _{t \rightarrow+\infty} \frac{1}{t} \int_{0}^{t} x(s) d s=\frac{G_{1}-\tilde{G}_{1}}{G}=0.5691, \lim _{t \rightarrow+\infty} \frac{1}{t} \int_{0}^{t} y(s) d s=\frac{G_{2}-\tilde{G}_{2}}{G}=0.3371$, $\lim _{t \rightarrow+\infty} \frac{1}{t} \int_{0}^{t} z(s) d s=\frac{G_{3}-\tilde{G}_{3}}{G}=0.1605$

Figure 3 Let (4.1) hold and $\alpha_{1}=0.2, \alpha_{2}=0.2$, $\alpha_{3}=0.3$ such that $\lambda_{1} a_{11} \geq \lambda_{2} a_{21}+\lambda_{3} a_{31}$, $\lambda_{2} a_{22} \geq \lambda_{1} a_{12}+\lambda_{3} a_{32}$ and $\lambda_{3} a_{33} \geq \lambda_{1} a_{13}+\lambda_{2} a_{23}$ (Theorem 2.6). Then (1.2) is globally asymptotically stable.

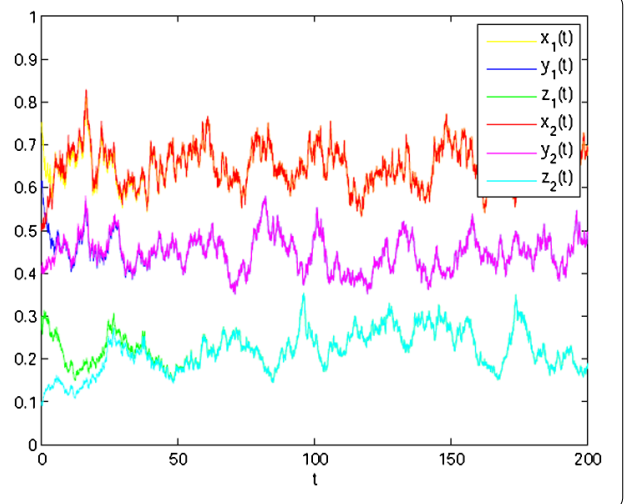

Table 1 The summary of the effect of the noise for species

\begin{tabular}{llll}
\hline Noise & Species & & \\
\cline { 2 - 4 } & $\boldsymbol{x}$ & $\boldsymbol{y}$ & $\boldsymbol{z}$ \\
\hline$\alpha_{1}$ & negative & negative & positive \\
$\alpha_{2}$ & negative & negative & positive \\
$\alpha_{3}$ & positive & positive & negative \\
\hline
\end{tabular}


Figure 4 Let (4.1) hold and $\alpha_{1}=0.3, \alpha_{2}=0.3$, $\alpha_{3}=0.2$. Note that $\delta_{1}=1.1, \delta_{2}=1.05, \delta_{3}=0.65$ and $x^{*}=G_{1} / G=0.6641, y^{*}=G_{2} / G=0.4648$, $z^{*}=G_{3} / G=0.3086$, we conclude that (2.2) holds. It follows from Theorems 2.5 and 2.7 that $\lim _{t \rightarrow+\infty} \frac{1}{t} x$ $\int_{0}^{t} x(s) d s=\int_{\mathbb{R}_{+}^{3}} \omega_{1} \mu\left(d \omega_{1}, d \omega_{2}, d \omega_{3}\right)=\frac{G_{1}-\tilde{G}_{1}}{G}$ a.s., $\lim _{t \rightarrow+\infty} \frac{1}{t} \int_{0}^{t} y(s) d s=\int_{\mathbb{R}_{+}^{3}} \omega_{2} \mu\left(d \omega_{1}, d \omega_{2}, d \omega_{3}\right)=$ $\frac{G_{2}-\tilde{G}_{2}}{G}$ a.s., $\lim _{t \rightarrow+\infty} \frac{1}{t} \int_{0}^{t} z(s) d s=\int_{\mathbb{R}_{+}^{3}} \omega_{3} \mu\left(d \omega_{1}, d \omega_{2}\right.$, $\left.d \omega_{3}\right)=\frac{G_{3}-\tilde{G}_{3}}{G}$ a.s.
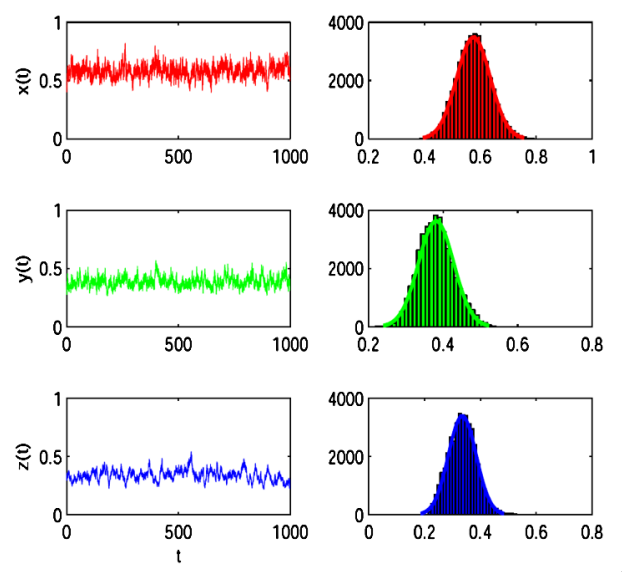

\section{Acknowledgements}

This work is supported by the National Natural Science Funds (No. 11772185), the National High Technology Research and Development Program of China (No. 2013AA122904), the Fundamental Research Funds for the Central Universities (No. HEUCFP201770), the Natural Science Foundation of Heilongjiang Province (No. F2015032), the Harbin Science and Technology Innovation Talent Youth Fund (No. RC2013QN001007).

\section{Competing interests}

The authors declare that they have no competing interests.

\section{Authors' contributions}

All authors drafted the manuscript, and they read and approved the final version of the manuscript.

\section{Author details}

${ }^{1}$ College of Automation, Harbin Engineering University, Harbin, China. ${ }^{2}$ College of Aerospace and Civil Engineering, Harbin Engineering University, Harbin, China. ${ }^{3}$ School of Mathematical Sciences, Heilongjiang University, Harbin, China.

\section{Publisher's Note}

Springer Nature remains neutral with regard to jurisdictional claims in published maps and institutional affiliations.

\section{Received: 8 October 2017 Accepted: 13 January 2018 Published online: 27 February 2018}

\section{References}

1. Liang, X, Jiang, JF: The dynamical behavior of type-K competitive Kolmogorov systems and its application to three-dimensional type-K competitive Lotka-Volterra systems. Nonlinearity 16, 785-801 (2003)

2. Wang, YS, Wu, H: Dynamics of a cooperation-competition model for the WWW market. Physica A 339, 609-620 (2004)

3. Jiang, JF, Cheng, ZX: The complete strategic classification for a cooperation-competition model in the WWW market. Physica A 363, 527-536 (2006)

4. Wang, QR, Zhou, K: Traveling wave solutions in delayed reaction-diffusion systems with mixed monotonicity. J. Comput. Appl. Math. 233, 2549-2562 (2010)

5. Xu, XL, Fu, CH, Chang, H, He, DR: An evolution model of complex systems with simultaneous cooperation and competition. Physica A 390, 3719-3726 (2011)

6. Liu, YB, Weng, PX: Asymptotic spreading of a three dimensional Lotka-Volterra cooperative-competitive system. Discrete Contin. Dyn. Syst., Ser. B 20, 505-518 (2015)

7. Aguirre, P, González-Olivares, E, Torres, S: Stochastic predator-prey model with Allee effect on prey. Nonlinear Anal., Real World Appl. 14, 768-779 (2013)

8. Ji, CY, Jiang, DQ: Dynamics of a stochastic density dependent predator-prey system with Beddington-DeAngelis functional response. J. Math. Anal. Appl. 381, 441-453 (2011)

9. Mandal, PS, Banerjee, M: Stochastic persistence and stability analysis of a modified Holling-Tanner model. Math. Methods Appl. Sci. 36, 1263-1280 (2013)

10. Saha, T, Chakrabarti, C: Stochastic analysis of prey-predator model with stage structure for prey. J. Appl. Math Comput. 35, 195-209 (2011)

11. Vasilova, M: Asymptotic behavior of a stochastic Gilpin-Ayala predator-prey system with time-dependent delay. Math. Comput. Model. 57, 764-781 (2013)

12. Yagi, A, Ton, TV: Dynamic of a stochastic predator-prey population. Appl. Math. Comput. 218, 3100-3109 (2011)

13. Jovanović, M, Vasilova, M: Dynamics of non-autonomous stochastic Gilpin-Ayala competition model with time-varying delays. Appl. Math. Comput. 219, 6946-6964 (2013)

14. Li, X, Mao, X: Population dynamical behavior of non-autonomous Lotka-Volterra competitive system with random perturbation. Discrete Contin. Dyn. Syst., Ser. A 24, 523-593 (2009)

15. Lian, BS, Hu, SG: Asymptotic behaviour of the stochastic Gilpin-Ayala competition models. J. Math. Anal. Appl. 339, 419-428 (2008) 
16. Zhu, C, Yin, G: On competitive Lotka-Volterra model in random environments. J. Math. Anal. Appl. 357, 154-170 (2009)

17. Jiang, DQ, Ji, CY, Li, XY, O’Regan, D: Analysis of autonomous Lotka-Volterra competition systems with random perturbation. J. Math. Anal. Appl. 390, 582-595 (2012)

18. Yao, Q, Liu, M: Global asymptotic stability of stochastic competitive system with infinite delays. J. Appl. Math. Comput. 50, 93-107 (2016)

19. Ji, CY, Jiang, DQ, Liu, H, Yang, QS: Existence, uniqueness and ergodicity of positive solution of mutualism system with stochastic perturbation. Math. Probl. Eng. 2010, Article ID 684926 (2010)

20. Liu, M, Wang, K: Analysis of a stochastic autonomous mutualism model. J. Math. Anal. Appl. 402, 392-403 (2013)

21. Liu, M, Wang, K: Population dynamical behavior of Lotka-Volterra cooperative systems with random perturbations. Discrete Contin. Dyn. Syst., Ser. A 33, 2495-2522 (2013)

22. Liu, Q: Analysis of a stochastic non-autonomous food-limited Lotka-Volterra cooperative model. Appl. Math. Comput. $254,1-8(2015)$

23. Li, M, Gao, HJ, Sun, CF, Gong, YZ: Analysis of a mutualism model with stochastic perturbations. Int. J. Biomath. 08 Artocle ID 1550072 (2015)

24. Liu, Q, Chen, QM, Hu, YY: Analysis of a stochastic mutualism model. Commun. Nonlinear Sci. Numer. Simul. 29, 188-197 (2015)

25. Liu, M, Wang, K: Dynamics of a two-prey one-predator system in random environments. J. Nonlinear Sci. 23, 751-775 (2013)

26. Mandal, PS: Characterization of positive solution to stochastic competitor-competitor-cooperative model. Electron. J. Differ. Equ. 2013, Article ID 88 (2013)

27. Liu, XY, Wei, YQ: Dynamics of a stochastic cooperative predator-prey system with Beddington-DeAngelis functional response. Adv. Differ. Equ. 2016, Article ID 21 (2016)

28. Caraballo, T, Colucci, R, Han, XY: Semi-Kolmogorov models for predation with indirect effects in random environments. Discrete Contin. Dyn. Syst., Ser. B 21(7), 2129-2143 (2016)

29. Caraballo, T, Colucci, R, Han, XY: Predation with indirect effects in fluctuating environments. Nonlinear Dyn. 84(1), 115-126 (2016)

30. Liu, M, Mandal, PS: Dynamical behavior of a one-prey two-predator model with random perturbations. Commun Nonlinear Sci. Numer. Simul. 28, 123-137 (2015)

31. Xing, ZJ, Cui, HT, Zhang, JM: Dynamics of a stochastic intraguild predation model. Appl. Sci. 6(4), Article ID 118 (2016)

32. Zhang, QM, Jiang, DQ: The coexistence of a stochastic Lotka-Volterra model with two predators competing for one prey. Appl. Math. Comput. 269, 288-300 (2015)

33. Liu, M, Bai, CZ: Analysis of a stochastic tri-trophic food-chain model with harvesting. J. Math. Biol. 73, $597-625$ (2016)

34. May, RM: Stability and Complexity in Model Ecosystems. Princeton University Press, New Jersey (1973)

35. Braumann, CA: Variable effort harvesting models in random environments: generalization to density dependent noise intensities. Math. Biosci. 177-178, 229-245 (2002)

36. Gard, TC: Introductions to Stochastic Differential Equations. Dekker, New York (1998)

37. Evans, SN, Ralph, P, Schreiber, SJ, Sen, A: Stochastic population growth in spatially heterogeneous environments. J. Math. Biol. 66, 423-476 (2013)

38. Mao, XR, Marion, G, Renshaw, E: Environmental Brownian noise suppresses explosions in population dynamics. Stoch Process. Appl. 97, 95-110 (2002)

39. Klebaner, FC: Introduction to Stochastic Calculus with Applications. World Scientific, Singapore (2005)

40. Barbalat, I: Systems d'equations differentielles d'oscillations nonlineaires. Rev. Roum. Math. Pures Appl. 4, 267-270 (1959)

41. Has'minskii, RZ: Stochastic Stability of Differential Equations. Sijthoff \& Noordhoff, Rockville (1980)

42. Strang, G: Linear Algebra and Its Applications. Thomson Learning, Inc., London (1988)

43. Zhu, C, Yin, G: Asymptotic properties of hybrid diffusion systems. SIAM J. Control Optim. 46, 1155-1179 (2007)

44. Higham, D: An algorithmic introduction to numerical simulation of stochastic differential equations. SIAM Rev. 43 525-546 (2001)

\section{Submit your manuscript to a SpringerOpen ${ }^{\circ}$ journal and benefit from:}

- Convenient online submission

- Rigorous peer review

- Open access: articles freely available online

- High visibility within the field

- Retaining the copyright to your article

Submit your next manuscript at $>$ springeropen.com 